\title{
Revisão narrativa sobre a automutilação não suicida entre adolescentes
}

\author{
Mara Cristiane von Mühlen \\ Sheila Gonçalves Câmara
}

Resumo: Nas últimas décadas, pode se perceber o aumento dos comportamentos de automutilação na adolescência. Embora isso possa ser explicado pelo crescente interesse de pesquisadores e especialistas em estudo esse fenômeno, é notório que a automutilação não suicida, considerada até recentemente como um comportamento específico inerente a quadros patológicos graves, tem sido utilizada como uma estratégia de enfrentamento mal-adaptativa por um número expressivo de adolescentes pelo menos uma vez na vida ou até mesmo por todo o período da adolescência. Nesta revisão narrativa de educação continuada a automutilação não suicida será discutida em termos de prevalências, funções e fatores associados. Os resultados apontam expressivas prevalências de automutilação não suicida em âmbito mundial, ligada a diversas funções e fatores associados relativos a aspectos sociodemográficos, individuais de manejo de problemas e emoções e psicossociais relacionais.

Palavras-chave: Automutilação não suicida; adolescência; fatores associados.

\section{Narrative review on nonsuicidal self injury among adolescents}

Abstract: In recent decades, one can notice the increase in self-mutilation behaviors in adolescence. Although this can be explained by the growing interest of researchers and study specialists in this phenomenon, it is notorious that non-suicidal self-mutilation, considered until recently as a specific behavior inherent to severe pathological conditions, has been used as a strategy of poorly adaptive coping by an expressive number of adolescents at least once in life or even for the entire period of adolescence. In this narrative review of continuing education, non-suicidal self-mutilation will be discussed in terms of prevalence, functions and associated factors. The results indicate significant prevalences of non-suicidal self-mutilation worldwide, linked to various functions and associated factors related to sociodemographic, individual-- aspects of problem management and relational emotions and psychosocial aspects.

Keywords: Nonsuicidal self injury; adolescence; associated factors.

\section{Introdução}

A adolescência é considerada uma fase de transição entre a infância e vida adulta, que vai dos 10 aos 19 anos, caracterizada por diversas alterações físicas e psicológicas (World Health Organization [WHO], 2014). Contribui para a confusão de uma identidade ainda em formação o fato de que os adolescentes são tratados como crianças enquanto lhe são feitas exigências pertinentes à fase adulta (Pimentel, 2014). Entretanto, como preconiza a Organização Mundial da Saúde (WHO, 2014), adolescentes precisam de uma atenção específica, distinta das que crianças e adultos recebem.

Durante as últimas duas décadas, alguns progressos podem ser apontados no que diz respeito à forma como a adolescência tem sido abordada: (1) uma melhor compreensão do estado de saúde dos adolescentes; (2) crescente clareza sobre os determinantes subjacentes à morte, à doença, e aos comportamentos relacionados à saúde durante a adolescência; (3) base de evidências mais expressiva para intervenções e; (4) maior compromisso e ação de governos nacionais e organizações não governamentais (Dick \& Fegunson, 2015). Estudos têm abordado a adolescência sob várias perspectivas, incluindo saúde pública, direitos humanos e desenvolvimento socioeconômico (Dick \& Fegunson, 2015; Jimenez et al., 2007; Fundo das Nações Unidas para a Infância [UNICEF], 2011).

Contudo, embora haja destaque pelos pesquisadores no progresso que foi feito, alguns relatórios têm evidenciado uma carência de pesquisas nesse período específico do desenvolvimento humano, que afeta tanto o indivíduo, quanto as famílias e as comunidades. (Jimenez et al., 2007; UNICEF, 2011). Para isso, importa que se investigue os comportamentos adotados pelos adolescentes no processo de constituição da sua identidade.

Os comportamentos adotados e as condições de vida têm um sério impacto na saúde, na construção identitária e no desenvolvimento dos adolescentes, com efeitos devastadores sobre sua saúde enquanto adolescentes - o que poderá refletir na vida adulta (Dick \& Fegunson, 2015). Cerca de metade de todos os transtornos de saúde mental começa aos 14 anos, mas a maioria dos casos não é reconhecida e nem tratada, com sérias consequências para a saúde mental ao longo da vida (WHO, 2014). Nesse 
cenário, e a partir dele construído, tem se observado o aumento da automutilação não suicida (AMNS) entre os adolescentes. Adolescentes se envolvem em AMNS em uma frequência muito maior do que os pais e os cuidadores podem estimar (Durand \& McGuinness, 2015). Há estimativas mundiais de que $21 \%$ dos adolescentes se envolveram em comportamentos autolesivos pelo menos uma vez durante a vida (Plener et al., 2018; Whitlock et al., 2011; Whitlock, Prussien \& Pietrusza, 2015).

Diante do exposto, este trabalho buscou, por meio de uma revisão narrativa da literatura, apresentar e discutir questões voltadas à prevalência, às funções e aos fatores associados à AMNS entre adolescentes.

\section{Método}

Trata-se de uma revisão narrativa da literatura. Para Rother (2007), as revisões narrativas dizem respeito a "publicações amplas, apropriadas para descrever e discutir o desenvolvimento de um determinado assunto, sob o ponto de vista teórico ou contextual". Ainda segundo Rother (2007), "as revisões narrativas não informam as fontes de informação utilizadas, a metodologia para busca das referências, nem os critérios utilizados na avaliação e seleção dos trabalhos.

Os dados sobre a AMNS entre adolescentes foram levantados a partir de uma revisão de literatura do último decênio, com busca e seleção realizadas entre os meses de novembro de 2017 e abril de 2018, em bases de dados indexadas no portal EBSCO (Academic Search Complete, MEDLINE Complete e Psychology and Behavioral Sciences Collection), no Portal Capes (PsycInfo e Web of Science) e na Pubmed, por meio dos descritores: "adolescence" e "non suicidal self-injury". Dentro do escopo nacional, os descritores "automutilação não suicida" e "adolescência" foram utilizados como estratégia de busca no SciELO (Scientific Eletronic Library Online), PEPsic (Periódicos Eletrônicos em Psicologia) e LILACS (Literatura Latino-Americana e do Caribe em Ciências da Saúde) e Google Acadêmico.

\section{Apresentação e discussão dos resultados}

Inicialmente serão apresentadas algumas considerações sobre a adolescência e a prática de AMNS nela inserida, seguidas de prevalências, de funções e de fatores associados à AMNS.

\section{Considerações sobre a adolescência e a automutilação não suicida}

A adolescência pode ser percebida com um período de vulnerabilidade em que, na mesma medida em que há potencialidade de mudanças, há também possibilidades de desequilíbrio, ou seja, pode ser vivenciada saudável ou patologicamente (Silva \& Siqueira, 2017). Assim sendo, o adolescente pode adotar comportamentos mal-adaptativos, como a AMNS (Jorge, Queirós \& Saraiva, 2015).

Já estabelecida como um comportamento de risco dentro de ambientes clínicos e educacionais, a AMNS está rapidamente se tornando um problema na sociedade em geral (Plener et al., 2018). Dentro das escolas, a AMNS foi descrita como uma "crise escolar silenciosa", refletindo conhecimento insuficiente, confusão, falta de intervenções efetivas e a tendência de os adultos evitarem lidar diretamente com a questão (Plener et al., 2018; Victor et al., 2018).

Não é possível afirmar se os dados sobre AMNS indicam aumento de sua prevalência ou se isso se deve à maior divulgação devido à recente atenção da mídia (Silva \& Siqueira, 2017) e a um maior interesse de pesquisadores e especialistas (Plener et al., 2018). No entanto, escolas (Wan, Xu, Chen, Hu \& Tao, 2015), hospitais, institutos de saúde mental e relatórios clínicos sugerem que a AMNS entre adolescentes está aumentando (Victor et al., 2018).

A nomenclatura que diz respeito a tais comportamentos ainda está em processo de construção, tanto no que diz respeito à definição como à busca do consenso dentro da literatura vigente, pois diversas são as formas encontradas dentro da literatura de nomear a prática de AMNS (Silva \& Siqueira, 2017). Por exemplo, alguns termos alternativos para AMNS são autodano deliberado, autolesão, automutilação e parasuicídio (Durand \& McGuinness, 2015; Muehlenkamp \& Brausch, 2012; Nock, 2010). Em estudo de revisão, Borges (2012) compilou diversos nomes utilizados, os quais apresentam diferenças em sua conceituação, como self-harm, self-injury e self-mutilation, enquanto que pesquisa realizada por Arcoverde e Soares (2012) mostrou que, em países com tradição em estudos sobre a AMNS como Inglaterra e Estados Unidos da América, é traçada uma diferenciação entre self-harm, self-injury e selfmutilation. Os termos self-harm e self-injury são comumente associados a lesões de menor extensão ou gravidade enquanto que self-mutilation está associado a lesões mais graves e extensas como ferimentos desfigurantes, amputações etc. (Arcoverde \& Soares, 2012). 
Diferenciações também são encontradas quanto à finalidade do comportamento, deliberate selfharm (autolesão deliberada), non suicidal self-injury (autolesão não suicida), sendo, deliberate self-harm relacionada a todo tipo de autolesão em que se reconhece a dificuldade na definição da intencionalidade do comportamento, e non suicidal self-injury relacionada à autolesão focada apenas na destruição do tecido corporal, sem intenção suicida, ainda incluindo o tipo da autolesão como cortes e outros comportamentos associados (queimaduras, arranhões, etc.) (Guerreiro \& Sampaio, 2013). No Brasil, a terminologia comumente utilizada é AMNS para todo o tipo de comportamento autolesivo, de acordo com pesquisa de revisão realizada por Silva e Siqueira (2017).

Neste estudo será considerada a AMNS como um ato deliberado de danificar físicamente o próprio tecido corporal sem intenção suicida, conforme preconiza a International Society for the Study of Self-Injury (2018), excluindo-se os comportamentos socialmente aceitos, como tatuagens e piercings, por exemplo (Nock, 2010). Entre as formas mais comuns de AMNS estão corte de pele, arranhões leves e severos, morder-se, bater em si mesmo, bater a cabeça na parede e puxar os próprios cabelos (Muehlenkamp \& Brausch, 2012; Nock, 2010). A AMNS acontece, geralmente, em cenário privado, uma vez que se encontra associada a um forte sentimento de vergonha (Siqueira \& Silva, 2017).

\section{Prevalências}

A seriedade da AMNS se tem evidenciado a partir de pesquisas que apontam altas prevalências entre o público adolescente de vários países. Isso pode ser verificado na Tabela 1.

Tabela 1

Prevalência de AMNS entre adolescentes

\begin{tabular}{|c|c|c|c|}
\hline País & Participantes (n) & Prevalência (\%) & Autor/ano \\
\hline Áustria, Alemanha e Suíça & 1.339 & 18,8 & Plener et al. (2013) \\
\hline \multirow[t]{3}{*}{ China } & 4.782 & 15 & You e Leung (2012 \\
\hline & 17.622 & 17 & Wan et al. (2015). \\
\hline & 2.317 & $\begin{array}{l}19,7 \text { em meninas } \\
13,4 \text { em meninos }\end{array}$ & Cheung et al. (2013) \\
\hline \multirow[t]{5}{*}{ EUA } & 284 & 17 & Muehlenkamp e Brausch, (2012) \\
\hline & 373 & 21,1 & Braush e Gutierrez (2010 \\
\hline & 12.068 & 27,6 & Brunner et al. (2013) \\
\hline & 150 & 30,7 & $\begin{array}{l}\text { Claes, Houben, Vandereycken, Bijttebier e Muehlenlamp } \\
\text { (2010). }\end{array}$ \\
\hline & 211 & 33,3 & Howe-Martin, Murrel e Guarnaccia (2012) \\
\hline Finlândia & 4.109 & 10,7 & Laukkanen et al. (2013) \\
\hline Portugal & 782 & $21,7 \%$ & Xavier, Gouveia e Cunha (2016) \\
\hline Suécia & 3.060 & $35,6 \%$ & Zetterqvist, Lundh, Dahlström e Svedin (2013) \\
\hline \multirow[t]{2}{*}{ Turquia } & 4.957 & $14,38 \%$ & Evren, Evren, Bozkurt e Can (2014) \\
\hline & 1.676 & $29 \%$ & Somer et al. (2015) \\
\hline
\end{tabular}

Fonte: dados da pesquisa

Percebe-se que são altas e preocupantes as prevalências em estudos que abrangem diversas origens. Apesar de terem sido encontrados estudos com prevalências menores, estas não descaracterizam a gravidade da prática entre os adolescentes. Válido assinalar que a verdadeira prevalência de AMNS entre adolescentes permanece desconhecida, pois esse comportamento é muitas vezes escondido e difícil de identificar, podendo estar associadas a diversas funções. O conteúdo da Tabela 1 sugere que apesar das diferenças culturais, sociais e econômicas, a AMNS é um problema de saúde pública que ultrapassa barreiras sociais e divisas demográficas. É geralmente aceito que a AMNS se tornou um problema crescente entre os adolescentes. As taxas de prevalência relatadas de AMNS entre adolescentes variam amplamente entre os estudos, não apenas devido a diferenças reais na prevalência em diversas culturas ou regiões, mas também, a diferentes definições e operacionalizações, o que deve ser considerado em pesquisas futuras.

\section{Funções da automutilação não suicida}

Caracterizada por um comportamento diferenciado da tentativa de suicídio, a AMNS denota um cunho de significativa dor emocional (Nock, 2010). Por meio da AMNS, o adolescente busca alívio imediato diante de um evento que lhe desperte inúmeros afetos negativos, para os quais não encontra 
outra via de expressão (Muehlenkamp \& Brausch, 2012; Nock, 2010). Logo após a AMNS, o adolescente sente alívio, um sentimento instantâneo e imediato que ocasiona a liberação da endorfina, o que acaba por diminuir a tensão que comumente antecede à prática (Somer et al., 2015).

A AMNS se dá depois de um evento estressor e é uma estratégia utilizada para lidar com a raiva sentida naquele momento pelo adolescente, ou seja, os adolescentes que se ferem de forma intencional relatam que a AMNS traz alívio da angústia emocional (Guerreiro \& Sampaio, 2013; Nock, 2010). Para além de uma forma de exprimir raiva, a AMNS pode ocorrer pela necessidade de se autopunir, produzir sensações normais ou até mesmo por distração (Nock, 2010). Entretanto, há de se compreender esse comportamento como uma forma de recuperar o controle da angústia gerada pelos afetos negativos.

Por meio de estudos de autorrelato realizados entre adolescentes, Muehlenkamp, Brausch, Quigley e Whitlock (2013) comprovaram que algumas das funções interpessoais dessa conduta são o "querer mostrar / comunicar desespero"; "tentar encaixar-se ou sentir-se perto de alguém"; "para ver se alguém me ama".

As razões pelas quais as pessoas se ferem diferem de uma para outra e múltiplas motivações podem coexistir (Nock, 2010). No entanto, parece que a AMNS é mais frequentemente motivada pela necessidade de regular os afetos negativos (Muehlenkamp et al., 2013). Nesse sentido, há evidências consideráveis de que a regulação emocional pode ser a principal função, acompanhada de vergonha e isolamento social (Nock, 2010). Assim, o indivíduo pode sentir-se preso em um ciclo doloroso de excitação emocional negativa e AMNS (Jutengren, Kerr \& Stattin, 2011). Diante de um quadro de regulação emocional, melhoras na regulação emocional estão refletidas na cessação da AMNS (Whitlock et al., 2015).

Mckenzie e Gross (2014) defendem que, no processo de regulação emocional, cinco estratégias podem direcionar os sujeitos à AMNS: (1) como uma alternativa para situações mais angustiantes; (2) seu ambiente social; (3) para desviar sua atenção de emoções desagradáveis ou pensamentos negativos; (4) para mudar as cognições sobre si mesmo por meio da autopunição; e, (5) para a mudança da consciência de si mesmo. A partir dessas evidências, a regulação da emoção desempenha um papel fundamental nas conceituações teóricas e no tratamento da AMNS, conforme preconizam Hasking Whitlock, Voon e Rose (2016) e Nock (2010).

Para alguns adolescentes que se automutilam, este comportamento também pode ser motivado pelo desejo de sentir-se mais vivo em resposta ao entorpecimento emocional ou para interromper os estados de dissociação ou desrealização (Nock, 2010). Também foi sugerido que a função de autopunição pode ser uma maneira de evitar as punições de outras pessoas, maximizando assim um senso de controle (Brown \& Plener, 2017; Durand \& McGuinness, 2015; Guerreiro, Sampaio \& Figueira, 2014; Muehlenkamp et al., 2013; Xavier et al., 2016). Para Nock (2010), a AMNS pode ser usada para comunicar angústia, induzir cuidados ou atenção dos outros, ou, na minoria dos casos, manipular as pessoas a se comportarem de maneira que não fariam. Nock (2010) afirmou que este comportamento autodestrutivo sem intencionalidade suicida pode ser repetido após uma primeira ocorrência, pois é uma forma eficaz de comunicar, influenciar e conectar com outras pessoas em diferentes contextos de vida. Ocorre, sobretudo, quando as tentativas menos extremas de comunicação não oferecem resultados positivos (Nock et al., 2008). Entre os adolescentes, a AMNS também pode fornecer uma maneira de as pessoas se relacionarem com os amigos que se automutilam (Muehlenkamp et al., 2013).

Embora, por definição, a AMNS não tenha a intenção de causar danos letais, as pessoas que adotam esse comportamento têm risco aumentado de lesão física grave ou morte (Koenig et al., 2017). Mesmo que o resultado da AMNS não seja tão devastador quanto o suicídio, ainda assim, precisa ter um espaço de importância e acolhimento

(Durand \& McGuinNess, 2015). Associados a esse comportamento, diversos são os fatores de risco encontrados na literatura.

\section{Fatores de risco para automutilação não suicida}

A prática da AMNS pode estar associada a diversos fatores de risco. A literatura tem evidenciado que estão associados a essa prática fatores sociodemográficos, individuais de manejo de problemas e de emoções e, psicossociais relacionais (Tabela 2).

Tabela 2

Fatores associados à AMNS entre adolescentes

\begin{tabular}{lll}
\hline Aspectos & Fatores & Autores/ano \\
\hline Sociodemográficos & Minorias sexuais & Batejan, Jarvi e Swenson, (2015) \\
\hline
\end{tabular}




\begin{tabular}{|c|c|c|}
\hline & Ser menina & $\begin{array}{l}\text { Cheung et al. (2013) } \\
\text { Giletta e et al. (2013) } \\
\text { Moran et al. (2015) } \\
\text { Voon, Hasking e Martin, (2014) } \\
\text { Zetterqvist et al. (2013) }\end{array}$ \\
\hline & Idade entre 13 e 15 anos & $\begin{array}{l}\text { Brunner et al. (2013) } \\
\text { Howe-Martin et al. (2012) }\end{array}$ \\
\hline \multirow{9}{*}{$\begin{array}{l}\text { Individuais de manejo de problemas e de } \\
\text { emoções }\end{array}$} & Altos níveis de afetos negativos & Van Vliet e Kalnins (2011) \\
\hline & Dificuldade de regular afeto & $\begin{array}{l}\text { Buser, Peterson e Kearney (2015) } \\
\text { Guerreiro et al. (2014) } \\
\text { Garreto (2015) }\end{array}$ \\
\hline & Estratégias de coping evitativas & $\begin{array}{l}\text { Guerreiro, Figueira, Cruz e Sampaio (2015) } \\
\text { Guerreiro et al. (2014) }\end{array}$ \\
\hline & $\begin{array}{l}\text { Estratégias de enfrentamento primárias e } \\
\text { inflexíveis }\end{array}$ & Somer et al. (2015) \\
\hline & Estratégias mal-adaptativas & \\
\hline & Repertório limitado de enfrentamento & $\begin{array}{l}\text { Buser et al. (2015) } \\
\text { Guerreiro et al. (2014) } \\
\text { Garreto (2015) } \\
\text { Van Vliet e Kalnins (2011) }\end{array}$ \\
\hline & $\begin{array}{l}\text { Descontentamento espiritual reforçado pela } \\
\text { crença de ser abandonado e ignorado por } \\
\text { Deus }\end{array}$ & Buser, Buser e Rutt (2017) \\
\hline & Educação cristã & Kress Newgent, Whitlock e Mease. (2015) \\
\hline & Autocrítica elevada & Buser et al. (2015) \\
\hline \multirow[t]{11}{*}{ Psicossociais relacionais } & Estresse interpessoal com pais e colegas & $\begin{array}{l}\text { Guerreiro et al. (2014) } \\
\text { Hawton, Saunders e O'Connor (2012) } \\
\text { Jutengren et al. (2011) }\end{array}$ \\
\hline & $\begin{array}{l}\text { Bullying pelos pares nas suas formas física, } \\
\text { verbal e relacional }\end{array}$ & $\begin{array}{l}\text { Klomek et al. (2016); Christoffersen, Mohl, } \\
\text { DePannfilis e Vammen. (2015) } \\
\text { Guerreiro et al. (2014) }\end{array}$ \\
\hline & $\begin{array}{l}\text { Incapacidade de implementar habilidades } \\
\text { sociais assertivas }\end{array}$ & $\begin{array}{l}\text { Guerreiro et al. (2014) } \\
\text { Guerreiro et al. (2015) }\end{array}$ \\
\hline & Influência dos pares & Prinstein et al. (2010) \\
\hline & $\begin{array}{l}\text { Contato mais frequente com métodos } \\
\text { específicos }\end{array}$ & $\begin{array}{l}\text { Guerreiro et al. (2015) } \\
\text { Zhu et al. (2016) }\end{array}$ \\
\hline & Maior exposição da AMNS na mídia & Zhu et al. (2016) \\
\hline & Baixo suporte social & $\begin{array}{l}\text { Brown et al. (2018) } \\
\text { Christoffersen et al. (2015) } \\
\text { Demirci (2018) } \\
\text { Guerreiro et al. (2014) } \\
\text { Martin, Raby, Labella e Roisman (2017) }\end{array}$ \\
\hline & $\begin{array}{l}\text { Sentimento de alienação em relação aos } \\
\text { pais }\end{array}$ & $\begin{array}{l}\text { Buser et al. (2015) } \\
\text { Yates, Tracy e Luthar (2008) }\end{array}$ \\
\hline & Relações familiares precárias & $\begin{array}{l}\text { Hawton et al. (2012) } \\
\text { Laukkanen et al. (2009) }\end{array}$ \\
\hline & Sentimento de abandono e de rejeição & Plener et al. (2013) \\
\hline & Maus-tratos na infância & $\begin{array}{l}\text { Brown et al. (2018) } \\
\text { Christoffersen et al. (2015) } \\
\text { Demirci (2018) } \\
\text { Guerreiro et al. (2014) } \\
\text { Martin et al. (2017) }\end{array}$ \\
\hline
\end{tabular}

Fonte: dados da pesquisa

A AMNS expressa sofrimento e mal-estar psicológico, acompanhados por falta de esperança, incapacidade de lidar com as emoções, falta de sensação de pertencimento e dificuldade de manter um sentimento de bem-estar psicológico. (Guerreiro \& Sampaio, 2013). A AMNS é considerada uma prática que precisa ser compreendida em toda sua complexidade, em que os adolescentes requerem entendimento e acompanhamento com o objetivo de lhes oportunizar um desenvolvimento saudável (Guerreiro et al., 2014; Muehlenkamp et al., 2013). 
Estudos longitudinais explicam que eventos negativos da vida e severa disciplina parental (Liu et al., 2014) predizem a AMNS. Além disso, o apoio parental percebido de forma reduzida prediz longitudinalmente o início da AMNS. Assim, as evidências existentes apoiam o vínculo entre o estresse entre os filhos e seus pais e a AMNS (Miller et al., 2018).

A inserção num grupo de pares em que esses comportamentos são divulgados e difundidos pode ter como consequência a experimentação de uma estratégia que alguém já experimentou como eficaz e que poderá contribuir para uma maior aceitação pelo grupo. $\mathrm{O}$ contágio e o modelamento social são fatores de risco para o início da utilização de comportamentos autolesivos, constituindo a escola um contexto em que essa difusão pode ocorrer (Jarvi, Jackson, Swenson \& Crawford, 2013).

E, embora se encontre em nível internacional um volume expressivo de pesquisas que contribui para que se possa decodificar as funções e correlações das manifestações de comportamento de AMNS, em termos de pesquisa nacionais existem lacunas que demonstram a necessidade de mais estudos para que se possa ter ciência da situação dos adolescentes quanto a esse tipo de comportamento. Ainda que a gravidade e a letalidade da AMNS variem de indivíduo e população, seu vínculo com o suicídio, com o sofrimento psicológico e com outras formas de doença mental está bem documentado (Brown \& Plener, 2017; Muehlenkamp \& Brausch, 2012; Stallard et al., 2013; Wan et al., 2015) e aponta para a necessidade de maior compreensão das variáveis envolvidas para se pensar em possibilidades de intervenção, de prevenção e de promoção da saúde.

\section{Considerações finais}

Apesar do aumento das taxas referentes à prevalência de AMNS e da crescente conscientização sobre esse comportamento, pouca discussão tem ocorrido sobre as formas de prevenir e reagir a este fenômeno que ocorre entre os adolescentes. Os achados demonstraram que os adolescentes que tentam a AMNS podem estar em risco de desenvolver um comportamento habitual que atua como habilidade temporária de enfrentamento e os coloca em maior risco de morte, uma vez que eles podem perder o controle sobre seu hábito.

Os achados também sugerem que os indivíduos que se automutilam não representam um único grupo homogêneo, mas a existência de segmentos menores, subgrupos homogêneos, de que indivíduos que praticam a AMNS compartilham traços em comum (motivação para esse comportamento, por exemplo). A implicação disso para a prática é que é importante avaliar uma ampla gama de comportamentos de AMNS, tanto indiretos quanto diretos.

Para além de sugestões mais abrangentes, pode-se pensar em intervenções nas escolas, espaço em que os adolescentes passam a maior parte do tempo e que contemplem os diversos fatores associados que se evidenciaram nesta pesquisa como, por exemplo: (1) criar políticas de procedimentos para todos nas escolas; (2) proporcionar educação aos funcionários de todos os níveis; (3) ações em sala de aula com foco na regulação emocional e habilidades de enfrentamento; (4) grupos de discussões sobre a AMNS e seus fatores de risco, envolvendo pais/responsáveis, escola e alunos, abrindo um canal de comunicação; (5) orientação em sala de aula, voltada a discutir a AMNS, suas feridas e cuidados físicos e alertar sobre os riscos de mortes acidentais; e, (6) reuniões com os adolescentes com AMNS para determinar a função do comportamento e verificar a necessidade de um atendimento terciário ou se as intervenções breves serão suficientes.

Apesar de sua relevância, o estudo apresenta limitações metodológicas, por se tratar de uma revisão narrativa que não permite sua reprodução. Mais estudos são necessários para ampliar o escopo do que aqui foi apresentado.

\section{Referências}

Arcoverde, R. L., \& Soares, L. S. L. C. (2012). Funções neuropsicológicas associadas a condutas autolesivas: revisão integrativa de literatura. Psicologia: Reflexão e Crítica, 25(2), 293-300.

Batejan, K. L., Jarvi, S. M., \& Swenson, L. P. (2015). Sexual orientation and non-suicidal self-injury: a meta-analytic review. Archives of Suicide Research, 19(2), 131-150.

Borges, C. N. L. O. (2012). À flor da pele: algumas reflexões a propósito de um estudo de caso sobre autolesão. Dissertação Mestrado. Instituto Universitário de Psicologia Aplicada. Lisboa, 2012. $\begin{array}{llllll}\text { Recuperado } & \text { em } & \text { de } & \text { Janeiro, }\end{array}$ <http://repositorio.ispa.pt/bitstream/10400.12/2282/1/14892.pdf>.

Brausch, A. M., \& Gutierrez, P. M. (2010). Differences in non-suicidal self-injury and suicide attempts in adolescents. Journal of Youth \& Adolescence, 39(3), 233-242. 
Brown, R. C., Heines, S., Witt, A., Braehler, E., Fegert, J. M., Harsch, D., \& Plener, P. L. (2018). The impact of child maltreatment on non-suicidal self-injury: data from a representative sample of the general population. BMC Psychiatry, 18(1), 181.

Brown, R., \& Plener, P. (2017). Non-suicidal self-injury in adolescence. CurrPsychiatryRep 19(20), 1-8.

Brunner, R., Kaess, M., Parzer, P., Fischer, G., Carli, V., Hoven, C. W., ... Wasserman, D. (2013). Lifetime prevalence and psychosocial correlates of adolescent direct self-injurious behavior: A comparative study of findings in 11 European countries. Journal of Child Psychology and Psychiatry, 55(4), 337-348.

Buser, J. K., Buser, T. J., \& Rutt, C.C. (2017). Nonsuicidal self-injury and spiritual/religious coping. Journal of Mental Health Counseling, 39 (2), 132-148.

Buser, T. J., Peterson, C. H., \& Kearney, A. (2015). Self-efficacy pathways between relational aggression and non-suicidal self-injury. Journal of College Counseling, 18(3), 195-208.

Cheung, Y., Wong, P., Lee, A., Lam, T. H., Fan, Y. S. S., \& Yip, P. (2013). Non-suicidal selfinjury and suicidal behavior: prevalence, co-occurrence, and correlates of suicide among adolescents in Hong Kong. Social Psychiatry \& Psychiatric Epidemiology, 48 (7), 11331144.

Christoffersen, M. N., Møhl, B., DePanfilis, D., \&Vammen, K. S. (2015). Non-suicidal self-injury-does social support make a difference? An epidemiological investigation of a Danish national sample. Child Abuse and Neglect, 44, 106-116.

Claes, L., Houben, A., Vandereycken, W., Bijttebier, P., \& Muehlenkamp, J. (2010). Brief report: The association between non-suicidal self-injury, self-concept and acquaintance with self-injurious peers in a sample of adolescents. Journal of Adolescence, 33(5), 775-778.

Demirci, E. (2018). Non-suicidal self-injury, emotional eating and insomnia after child sexual abuse: Are those symptoms related to emotion regulation? Journal of Forensic and Legal Medicine, 53, 17-21.

Dick, B., \& Fergunson, J. B. (2015). Health for the world's adolescents: a second chance in the second decade. Journal of Adolescent Health 56, 3-6.

Durand, S. C., \& McGuinness, T. M. (2015). Adolescents who self-injure. Journal of Psychosocial Nursing and Mental Health Services, (4), 26-29.

Evren, C., Evren, B., Bozkurt, M., \& Can, Y. (2014). Non-suicidal self-harm behavior within the previous year among 10th-grade adolescents in Istanbul and related variables. Nordic Journal of Psychiatry, 68(7), 481-487.

Fundo das Nações Unidas para a Infância (2011). The state of the world's children 2011. Adolescence: an age of opportunity. New York: United Nations Children's Fund. UNICEF. Recuperado em 05 de Novembro, 2017, de <https://www.unicef.org/sowc2011/>.

Garreto, A. K. R. (2015). O desempenho executivo de pacientes que apresentam automutilação. $112 \mathrm{f}$. Dissertação. (Mestrado em Ciências) - Faculdade de Medicina da Universidade de São Paulo, São Paulo.

Giletta, M., Burk, W. J., Scholte, R. H. J., Engels, R. C. M. E., \& Prinstein, M. J. (2013). Direct and indirect peer socialization of adolescent nonsuicidal self-injury. Journal of Research on Adolescence (Wiley-Blackwell), 23(3), 450-463.

Guerreiro, D. F., \& Sampaio, D. (2013). Comportamentos autolesivos em adolescentes: uma revisão da literatura com foco na investigação em língua portuguesa. Revista Portuguesa de Saúde Pública, 31(2), 213-222.

Guerreiro, D. F., Figueira, M. L., Cruz, D., \& Sampaio, D. (2015). Coping strategies in adolescentes who self-harm: A community sample study. The Journal of Crisis Intervention and Suicide Prevention, 36(1), 31-37.

Guerreiro, D. F., Sampaio D., \& Figueira, M. L. (2014). Relatório de investigação "Comportamentos autolesivos em adolescentes: características epidemiológicas e análise de fatores psicopatológicos, temperamento afectivo e estratégias de coping". Clínica Universitária de Psiquiatria e Psicologia. Universidade de Lisboa. Recuperado em 19 fev. 2018, de: 〈http://www.spsuicidologia.pt/generalidades/biblioteca/143>.

Hasking, P., Whitlock, J., Voon, D., \& Rose, A. (2016). A cognitive-emotional model of NSSI: using emotion regulation and cognitive processes to explain why people self-injure. Cognition and Emotion, 31(8), 1543-1556.

Hawton, K., Saunders, K. E., \& O’Connor, R. C. (2012). Self-harm and suicide in adolescents. Lancet, 379(9834), 2373-2382. 
Howe-Martin, L. S., Murrell, A. R., \& Guarnaccia, C. A. (2012). Repetitive nonsuicidal self-injury as experiential avoidance among a community sample of adolescents. Journal of Clinical Psychology, 68(7), 809-829.

International Society for the Study of Self-Injury. (2018). Definition of non-suicidal self-injury. Recuperado em 19 fev, 2018, de: <http://www.itriples.org/isss-aboutself-i.html>.

Jarvi, S., Jackson, B., Swenson, L. \& Crawford, H. (2013). The impact of social contagion on nonsuicidal self-injury: a review of the literature. Archives of Suicide Research, 17, 1-19.

Jimenez, E. Y., Fares, J., Gauri, V., Lundberg, M. K. A., McKenzie, D., Murthi, M., Ridao-Cano, C., \& Sinha, N. (2007). World development report 2007: Development and the next generation. Washington, D.C: The World Bank. Recuperado em 05 de Novembro, 2017, de <http://documents.worldbank.org/curated/en/556251468128407787/pdf/359990WDR0complete.pdf $>$.

Jorge, J. C., Queirós, O., \& Saraiva, J. (2015). Descodificação dos comportamentos autolesivos sem intenção suicida: Estudo qualitativo das funções e significados na adolescência. Análise Psicológica, 33(2), 207-219.

Jutengren, G., Kerr, M., \& Stattin, H. (2011). Adolescents' deliberate self-harm, interpersonal stress, and the moderating effects of self-regulation: A two-wave longitudinal analysis. Journal of School Psychology, 49(2011) 249-264.

Koenig J, Rinnewitz L, Niederbäumer M, Strozyk T, Parzer P, Resch F, \& Kaess M. (2017). Binge/purge thoughts in non-suicidal self-injurious adolescents: an ecological momentary analysis. Journal of Psychiatric Research, 89, 81-84.

Klomek, A.B., Snir, A., Apter, A., Carli, V., Wasserman, C., Hadlaczky, G.... Wasserman, D. (2016). Association between victimization by bullying and direct self-injurious behavior among adolescence in Europe: a ten-country study. European Child and Adolescent Psychiatry, 25, 1183-1193.

Kress, V. E., Newgent, R. A., Whitlock, J., \& Mease, L. (2015). Spirituality/religiosity, life satisfaction, and life meaning as protective factors for nonsuicidal self-injury in college students. Journal of College Counseling, 18(2), 160-174.

Laukkanen, E., Rissanen, M-L., Honkalampi, K., Kylma, J. Tolmunen, T., \& Hintikka, J. (2009). The prevalence of self-cutting and other self-harm among 13- to 18-year-old Finnish adolescents. Social Psychiatry and Psychiatric Epidemiology, 44, 23-28.

Laukkanen, E., Rissanen, M-L., Honkalampi, K., Kylma, J. Tolmunen, T., \& Hintikka, J. (2013). Adolescent self-cutting elsewhere than on the arms reveals more serious psychiatric symptoms. European Child \& Adolescent Psychiatry, 22(8), 501-510.

Liu, R. T., Frazier, E. A., Cataldo, A. M., Simon, V. A., Spirito, A., \& Prinstein, M. J. (2014). Negative life events and non-suicidal self-injury in an adolescent Inpatient Sample. Archives of Suicide Research, 18(3), 251-258.

Martin, J., Raby, K. L., Labella, M. H., \& Roisman, G. I. (2017). Childhood abuse and neglect, attachment states of mind, and non-suicidal self-injury. Attachment \& Human Development, 19(5), 425-446.

McKenzie, K. C., \& Gross, J. J. (2014). Non-suicidal self-injury: an emotion regulation perspective. Psychopathology, 47(4), 207-219.

Mikolajczak, M., Petrides, K. V., \& Hurry, J. (2009). Adolescents choosing self-harm as an emotion regulation strategy: The protective role of trait emotional intelligence. British Journal of Clinical Psychology, 48 (2), 181-193.

Miller, A. B., Linthicum, K. P., Helms, S. W., Giletta, M., Rudolph, K. D., Hastings, P. D., Nock, M.K., \& Prinstein, M. J. (2018). Reciprocal associations between adolescent girls' chronic interpersonal stress and non-suicidal self-injury: a multi-wave prospective investigation. Journal of Adolescent Health, 63(6), 694-700.

Moran, P., Coffey, C., Romaniuk, H., Degenhardt, L., Borschmann, R., \& Patton, G. C. (2015). Substance use in adulthood following adolescent self-harm: a population-based cohort study. Acta Psychiatrica Scandinavica, 131 (1), 61-68.

Muehlenkamp, J. J., \& Brausch, A. M. (2012). Bodyimage as a mediator of non-suicidal selfinjury in adolescents. Journal of Adolescence, 35(1), 1-9.

Muehlenkamp, J., Brausch, A., Quigley, K., \& Whitlock, J. (2013). Interpersonal features and functions of non-suicidal self-injury. Suicide and Life-Threatening Behavior, 43(1), 67-80.

Nock, M. (2010). Self-injury. Annual Review of Clinical Psychology, 6, 339-63. 
Nock, M., Borges, G., Bromet, E. J., Cha, C. B., Kessler, R. C., \& Lee, S. (2008). Suicide and suicidal behaviour. Epidemiologic Reviews, 30, 133-154.

Pimentel, J. P. R. (2014). O adolescente marginalizado frente à modernidade líquida. Protestantismo em Revista, 33, 68-74.

Plener, P. L., Kaess, M., Schmahl, C., Pollak, S., Fegert, J. M., \& Brown, R. C. (2018). Nonsuicidal selfinjury in adolescents. Deutsches Aerzteblatt Online, 115(3), 23-30.

Plener, P., Fischer, C., In-Albon, T., Rollett, B., Nixon, M., Groschwitz, R., \& Schmid, M. (2013). Adolescent non-suicidal self-injury (NSSI) in German-speaking countries: comparing prevalence rates from three community samples. Social Psychiatry \& Psychiatric Epidemiology, 48(9), 1439-1445.

Prinstein, M. J., Heilbron, N., Guerry, J.D., Franklin, J. C., Rancourt, D., Simon, V., \& Spirito, A. (2010) Peer influence and non-suicidal self-injury: longitudinal results in community and clinicallyreferred adolescent samples. Journal of Abnormal Child Psychology, 38, 669-682.

Rother, E. T. (2007). Revisão sistemática X revisão narrativa. Acta Paulista de Enfermagem, 20(2), v-vi.

Silva, M. F. de A., \& Siqueira, A. C. (2017). O perfil de adolescentes com comportamentos de autolesão identificados nas escolas estaduais em Rolim de Moura - RO. Revista FAROL, 3(3), 5-20.

Somer, O., Bildik, T., Kabukçu-Başay, B., Güngör, D., Başay, Ö., \& Farmer, R. (2015). Prevalence of non-suicidal self-injury and distinct groups of self-injurers in a community sample of adolescents. Social Psychiatry \& Psychiatric Epidemiology. 50(7), 1163-1171.

Stallard, P., Spears, M., Montgomery, A. A. , Phillips, R., \& Sayal, K. (2013). Self-harm in young adolescents (12-16 years): onset and short-term continuation in a community 0-sample. BMC Psychiatry, 2(13), 328.

Van Vliet, K. J., \& Kalnins, G. R. C. (2011). A Compassion-Focused Approach to Nonsuicidal SelfInjury. Journal of Mental Health Counseling, 33(4), 295-311.

Victor, S. E., Muehlenkamp, J. J., Hayes, N. A., Lengel, G. J., Styer, D. M., \& Washburn, J. J. (2018). Characterizing gender differences in non-suicidal self-injury: evidence from a large clinical sample of adolescents and adults. Comprehensive Psychiatry, 82, 53-60.

Voon, D., Hasking, P., \& Martin, G. (2014). The roles of emotion regulation and ruminative thoughts in non-suicidal self-injury. British Journal of Clinical Psychology, 53, 95-113.

Wan, Y-H., Xu, S-J., Chen, J., Hu, C-L., \& Tao, F-B. (2015). Longitudinal effects of psychological symptoms on non-suicidal self-injury: a difference between adolescents and young adults in China. Social Psychiatry \& Psychiatric Epidemiology, 50(2), 237-247.

Whitlock, J., Muehlenkamp, J., Purington, A., Eckenrode, J., Barreira, P., Baral Adams, G., \& Knox, K. (2011). Nonsuicidal self-injury in a college population: general trends and sex differences. Journal of American College Health, 59, 691-698.

Whitlock, J., Prussien, K., \& Pietrusza, C. (2015). Predictors of self-injury cessation and subsequent psychological growth: results of a probability sample survey of students in eight universities and colleges. Child and Adolescent Psychiatry and Mental Health, 9(19), 1-12.

World Health Organization (2014). Health for the World's Adolescents: a second chance in the second decade. WHO. Recuperado de <http://apps.who.int/adolescent/second-decade/>.

Xavier, A., Gouveia, J.P., \& Cunha, M. (2016). Non-suicidal self-injury in adolescence: the role of shame, self-criticism and fear of self-compassion Child \& Youth Care Forum, 45(4), 571-586.

Yates, T. M., Tracy, A. J., \& Luthar, S. S. (2008). Nonsuicidal self-injury among "privileged"' youths: longitudinal and cross-sectional approaches to developmental process. Journal of Consulting and Clinical Psychology, 76(1), 52-62.

You, J., \& Leung, F. (2012). The role of depressive symptoms, family invalidation and behavioral impulsivity in the occurrence and repetition of non-suicidal self-injury in chinese adolescents: a 2year follow-up study Journal of Adolescence, 35(2), 389-395.

Zetterqvist, M., Lundh, L-G., Dahlström, Ö., \& Svedin, C. (2013). Prevalence and function of nonsuicidal self-injury (NSSI) in a community sample of adolescents, using suggested DSM-5 Criteria for a potential NSSI disorder. Journal of Abnormal Child Psychology, 41 (5), 59-773.

Zhu, L., Westers, N. J., Horton, S.E., King, J. D., Diederich, A., Stewart, S.M. \& Kennard, B. D. (2016). Frequency of exposure to and engagement in nonsuicidal self-injury among inpatient adolescents. Archives of Suicide Research, 20(4), 580-590. 
Mara Cristiane von Mühlen: Psicóloga, mestre em psicologia e saúde Universidade Federal de Ciências da Saúde de Porto Alegre. Rua Rodolfo Heller, 744, Bairro São João, Montenegro/RS- CEP: 95780-000 Sheila Gonçalves Câmara: Doutora em psicologia, Universidade Federal de Ciências da Saúde de Porto Alegre.

E-mail para contato: psicoulbra.mara@hotmail.com 